\title{
УРАВНЕНИЕ ДИНАМИКИ НЕОБРАТИМОЙ СОРБЦИИ ПЕРВОГО ПОРЯДКА НА ПРИМЕРЕ СОРБЦИИ АНТОЦИАНОВ БЕНТОНИТОВОЙ ГЛИНОЙ
}

\author{
(с) 2020 г. В. И. Дейнека ${ }^{a, *}$, А. Г. Доронин ${ }^{a}$, Л. А. Дейнека ${ }^{a}$ \\ ${ }^{a}$ Белгородский государственный национальный исследовательский университет, Белгород, Россия \\ *e-mail: deineka@bsu.edu.ru \\ Поступила в редакцию 17.02.2019 г. \\ После доработки 17.02.2019 г. \\ Принята к публикации 14.05.2019 г.
}

\begin{abstract}
Предложено новое уравнение для необратимой сорбции веществ по реакции первого порядка с учетом свободной поверхности сорбента взамен популярного уравнения Лагергрена. По предложенному уравнению со временем с начала сорбции линейно связан параметр $\ln \left(\frac{c_{\tau}}{c_{\tau}-c_{\text {кон }}}\right)$, в котором $c_{\tau}$ и $c_{\text {кон }}-$ текущая и конечная (при полном заполнении поверхности) концентрации сорбата соответственно. Показано, что предложенное уравнение эффективно описывает динамику сорбции антоцианов различного строения без исключения начальных экспериментальных точек, что характерно для использования уравнения Лагергрена.
\end{abstract}

Ключевые слова: новое уравнение, динамика сорбции, необратимая сорбция первого порядка, степень заполнения поверхности, антоцианы, бентонитовая глина

DOI: $10.31857 / \mathrm{S} 0044453720010057$

Одним из часто [1] используемых для анализа скорости сорбции веществ из жидкой фазы на твердых сорбентах является уравнение, выводимое в рамках приближения сорбции необратимой реакцией псевдо-первого порядка, предложенное Лагергреном, по простой модели:

$$
\frac{d Q_{\tau}}{d \tau}=k_{1}\left(Q_{e}-Q_{\tau}\right)
$$

где $\tau$ - время с начала сорбции, $Q_{\tau}-$ степень (лучше - уровень) заполнения поверхности сорбента в момент времени $\tau, k_{1}-$ константа скорости сорбции по псевдо-первому порядку, $Q_{e}-$ уровень заполнения поверхности при насыщении.

При интегрировании уравнения (1) получают удобное для графического построения и нахождения условной константы уравнение:

$$
\lg \left(Q_{e}-Q_{\tau}\right)=\lg \left(Q_{e}\right)-\frac{k_{1}}{2.303} \tau .
$$

Как отмечается в работе [2], уравнение (2) применимо к экспериментальным результатам и при отличии процесса от первого порядка. При этом параметр $\left(Q_{e}-Q_{\tau}\right)$ может не соответствовать числу свободных центров, а $Q_{e}$ не равен отрезку, отсекаемому прямой линией на графике по уравнению (2).

Выведение и использование уравнения (2) удивительно по той причине, что существенно более точное и надежное уравнение может быть предложено взамен данного, что и стало задачей настоящей работы.

\section{ЭКСПЕРИМЕНТАЛЬНАЯ ЧАСТЬ}

Антоцианы получали экстракцией $0.1 \mathrm{M}$ водным раствором $\mathrm{HCl}$ из растительных материалов. Полученные экстракты очищали сорбцией на бентонитовой глине, с которой антоцианы десорбировали смесью $0.1 \mathrm{M}$ водный раствор $\mathrm{HCl}-$ этанол в соотношении 1:1. Этанол отгоняли на вакуумном ротационном испарителе и остающийся раствор разбавляли $0.1 \mathrm{M}$ водным раствором $\mathrm{HCl}$ для исследования динамики сорбции.

В работе использовали бентонитовую глину Бентокон “Медиум” (ООО “Бентопром”).

При исследовании динамики сорбции к подготовленному раствору антоцианов в $0.1 \mathrm{M}$ водном растворе $\mathrm{HCl}$ объемом 150 мл добавляли навеску бентонитовой глины $(0.030-0.100$ г) и 


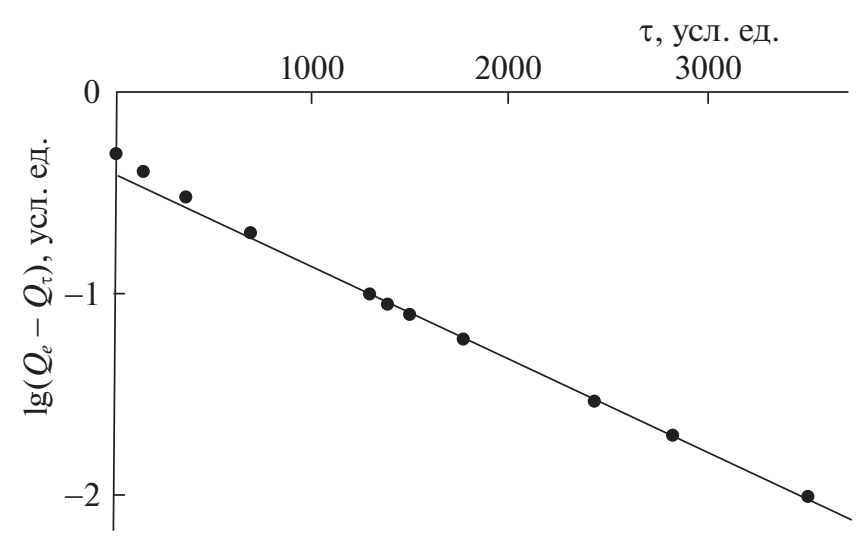

Рис. 1. Применение уравнения Лагергрена к расчетным данным при изменении концентрации сорбата в конечном состоянии в 1.5 раза.

включали перемешивание. Через каждые 5 мин отбирали аликвотные объемы смеси (5 мл), центрифугировали 3 мин, и определяли оптическую плотность раствора. Раствор с осадком возвращали в исходную смесь.

Концентрацию антоцианов определяли прямым спектрофотометрическим методом в кварцевых кюветах на приборе Shimadzu UV-1550 при pH 1 без дифференциального подхода [3], поскольку полимерные антоцианы отделялись на стадии очистки.

Для контроля видового состава антоцианов использовали хроматограф Agilent Infinity 1200 с диодно-матричным и масс-спектрометрическим детектированием. Колонка: $150 \times 2.1$ мм Kromasil $100-5 \mathrm{C} 18$, ионизацию осуществляли в режиме электроспрея при сканировании положительных ионов, напряжение на фрагменторе 225 В. Градиентный режим элюирования - элюент А: 6 об. \% $\mathrm{CH}_{3} \mathrm{CN}, 10$ об. \% НСООН в воде, элюент Б: 20 об. \% $\mathrm{CH}_{3} \mathrm{CN}, 10$ об. \% $\mathrm{HCOOH}$ в воде; программа: 0 мин $-100 \%$ А; 20 мин $100 \%$ Б; 21 мин $100 \%$ А, 30 мин - $100 \%$ А. Скорость подачи элюента 0.2 мл/мин.

\section{ОБСУЖДЕНИЕ РЕЗУЛЬТАТОВ}

Следуя Лагергрену, рассмотрим сорбцию веществ на твердой однородной поверхности по необратимой реакции, но с отказом от простого уравнения первого порядка. Так, скорость сорбции вещества должна быть пропорциональной концентрации вещества в фазе раствора, $c_{\tau}$, и количеству свободных мест на поверхности сорбента, $\theta_{\text {св. } \tau}$ :

$$
-\frac{d c_{\tau}}{d \tau}=k_{1} c_{\tau} \theta_{\text {св. } \tau}
$$

Связь между концентрацией вещества в растворе и количеством свободных мест на поверхности сорбента можно установить, вводя новую константу $k_{2}$, связывающую изменение концентрации вещества в растворе с изменением числа мест на этой поверхности, начальную $\left(c_{0}\right)$ и конечную (при которой произойдет насыщение поверхности, $c_{\text {полн }}$ ) концентрации вещества в растворе:

$$
\begin{gathered}
\theta_{\text {св. } \tau}=\theta_{\text {полн }}-\theta_{\text {зан. } \tau}=k_{2}\left(\Delta c_{\max }-\Delta c_{\tau}\right)= \\
=k_{2}\left[\left(c_{\text {нач }}-c_{\text {полн }}\right)-\left(c_{\text {нач }}-c_{\tau}\right)\right]=k_{2}\left(c_{\tau}-c_{\text {полн }}\right) .
\end{gathered}
$$

В таком случае уравнение (3) будет записано в виде:

$$
-\frac{d c_{\tau}}{d \tau}=k_{1} c_{\tau} k_{2}\left(c_{\tau}-c_{\text {полн }}\right) .
$$

Точное решение этого уравнения получаем интегрированием:

$$
-\int_{0}^{\tau} \frac{d c_{\tau}}{c_{\tau}\left(c_{\tau}-c_{\mathrm{KOH}}\right)}=\int_{0}^{\tau} k_{1} k_{2} d \tau .
$$

И это решение имеет вид:

$$
\frac{1}{c_{\mathrm{KOH}}} \ln \left(\frac{c_{\tau}}{c_{0}} \frac{c_{0}-c_{\text {Кон }}}{c_{\tau}-c_{\mathrm{KоH}}}\right)=k_{1} k_{2} \tau .
$$

В соответствие с полученным уравнением линейность следует искать в координатах $\ln \left(\frac{c_{\tau}}{c_{\tau}-c_{\text {кон }}}\right)$ относительно времени сорбции $\tau$ при контроле применимости предложенного в работе подхода:

$$
\begin{aligned}
& \ln \left(\frac{c_{\tau}}{c_{\tau}-c_{\text {кон }}}\right)=c_{\text {кон }} k_{1} k_{2} \tau+ \\
& +\ln \left(\frac{c_{0}}{c_{0}-c_{\text {кон }}}\right)=a \tau+b .
\end{aligned}
$$

С другой стороны, используя полученное уравнение, можно составить таблицу с расчетными временами сорбции при заданных концентрациях сорбируемого вещества в растворе и в сорбированном состоянии, что позволяет анализировать применимость к этим данным уравнения Лагергрена, рис. 1 и рис. 2. Первый вывод - уравнение действительно может быть применено, но только на качественном уровне (обнаружение линейности для некоторого участка на соответствующем графике), при этом:

- на начальном участке графика отклонения линии тренда от экспериментальных точек могут быть значительными (линия тренда смещена вниз от начальных точек); 


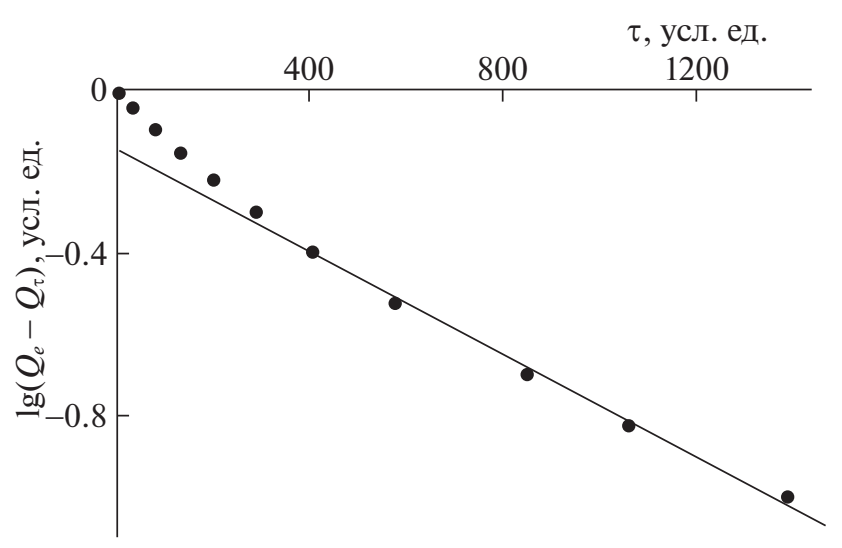

Рис. 2. Применение уравнения Лагергрена к расчетным данным при изменении концентрации сорбата в конечном состоянии в 3 раза.

- отклонения уменьшаются при сближении концентраций сорбата в начальном и конечном растворах. То есть метод применим при минимально возможном изменении концентрации раствора в результате сорбции. Однако следует учесть, что при этом требуется высокая точность определения концентраций сорбата;

- при высоком уровне снижения концентрации раствора при сорбции анализ по линейному уравнению Лагергрена нецелесообразен вообще.

В качестве модельного процесса рассмотрим сорбцию антоцианов на поверхности бентонитовой глины. Такая сорбция из 0.1 М водного раствора протекает практически необратимо (для десорбции антоцианов необходимо введение в раствор органического модификатора [4]). Ионообменный характер сорбции антоцианов [5] при этом обеспечивает мономолекулярную сорбцию, что удовлетворяет начальным условиям предлагаемой модели.

Рассмотрим сорбцию антоцианов экстракта плодов паслена садового (Solanum retroflexum L.), представленного в основном одним компонентом - петунидин-3-(6"'-napa-кумароилрутинозид)-5-глюкозидом [6]. Полученные результаты, действительно, показывают прямолинейную зависимость в координатах уравнения Лагергрена, но в ограниченном диапазоне экспериментальных данных. При этом начальные точки располагаются сушественно выше, как было предсказано выше линии тренда, а расположение конечных точек трудно считать надежным показателем изза большой экспериментальной ошибки в данном случае, рис. 3. В координатах предлагаемого в работе уравнения (8) получается хорошая прямолинейная зависимость без исключения начальных точек полученных в ходе эксперимента, рис. 4.

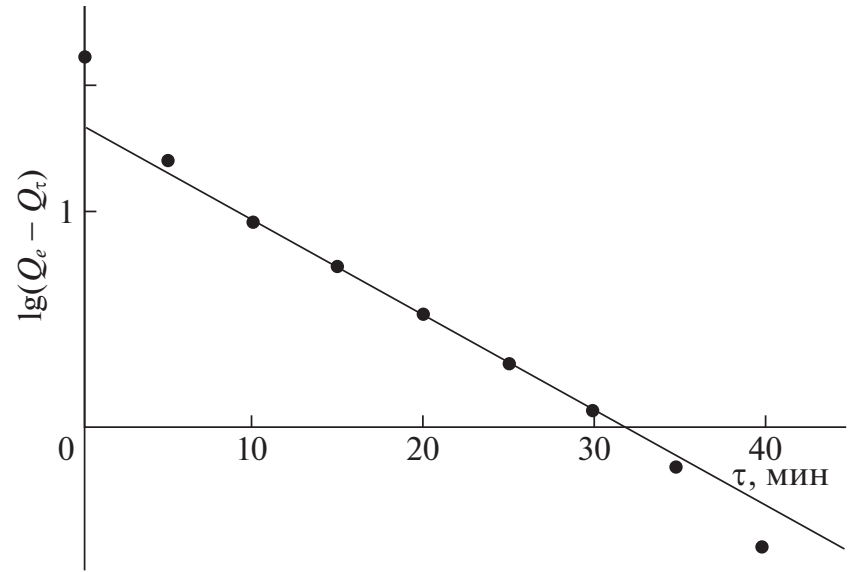

Рис. 3. Описание динамики сорбции антоцианов паслена садового на бентонитовой глине по уравнению Лагергрена при уменьшении концентрации антоцианов в конечном состоянии в 3 раза.

При этом, как и предыдущем случае при достижении конечного насыщения поверхности погрешности в определении концентрации также могут быть большими.

При сорбции антоцианов плодов аронии Мичурина (Aronia mitschurinii A.K. Skvortsov and Maitul., ошибочно принимаемой за аронию черноплодную), представленных в значительной степени цианидин-3-галактозидом [7], получены аналогичные данные. При этом в координатах предлагаемого уравнения (8) также получается прямолинейная зависимость без исключения начальных экспериментальных точек, рис. 5. Отметим, что и наклоны прямых на рис. 4 и рис. 5 имеют близкие значения, что при примерном равенстве начальных условий (по суммарной концентрации

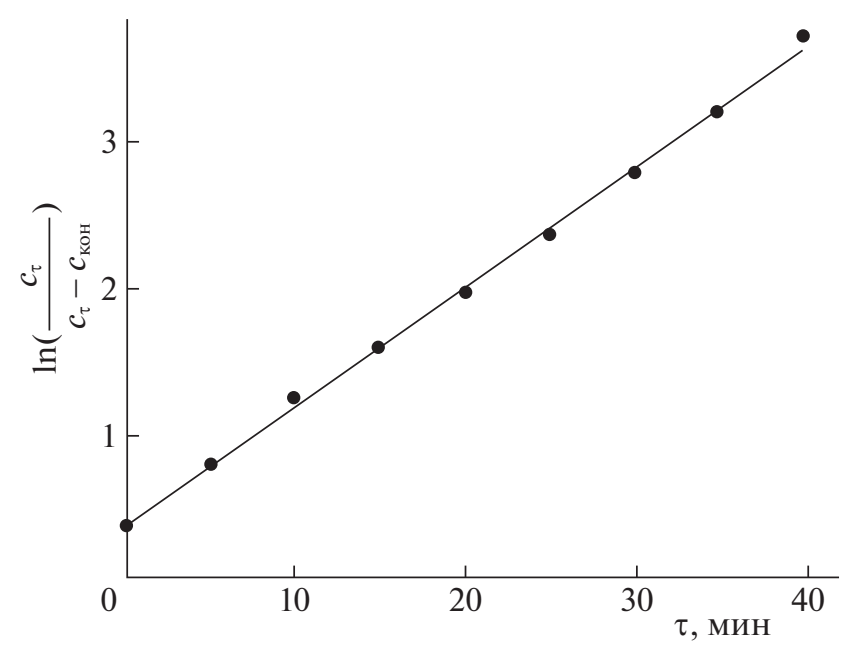

Рис. 4. Описание динамики сорбции антоцианов паслена садового на бентонитовой глине по уравнению (8) при уменьшении концентрации антоцианов в конечном состоянии в 3 раза. 


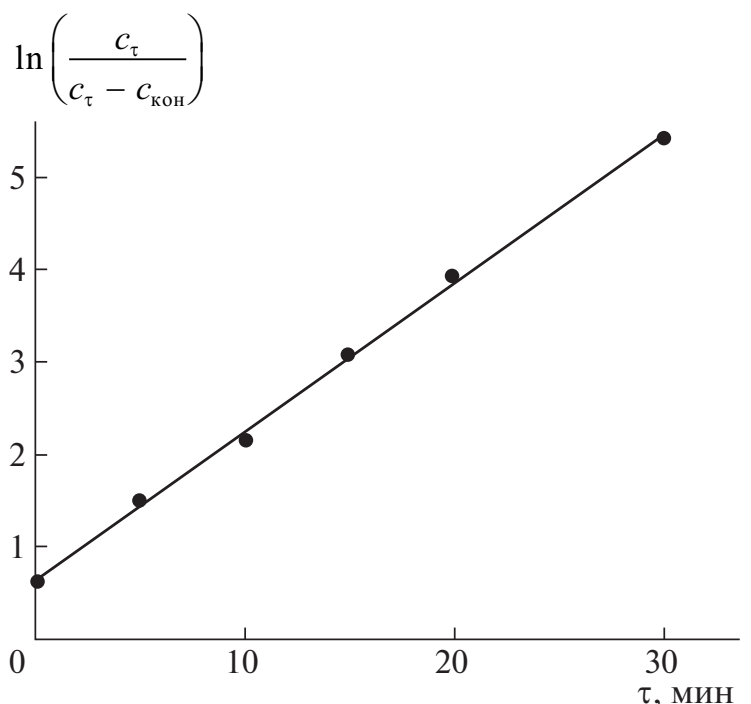

Рис. 5. Описание динамики сорбции антоцианов плодов аронии Мичурина на бентонитовой глине по уравнению (8) при уменьшении концентрации антоцианов в конечном состоянии в 3.5 раза.

антоцианов, массам навесок добавляемой бентонитовой глины и температуре) указывает на близость констант скоростей сорбции антоцианов с различной структурой. При сорбции антоцианов лепестков цветков розы, представленных цини- дин-3,5-диглюкозидом [8], также были получены результаты с прямолинейной зависимостью без исключения начальных точек.

Следовательно, предложенное уравнение (8) существенно лучше описывает динамику сорбции антоцианов на бентонитовой глине в предположении необратимой сорбции первого порядка с учетом степени заполнения поверхности глины по сравнению с уравнением Лагергрена.

\section{СПИСОК ЛИТЕРАТУРЫ}

1. Ho Y.-S. // Scientometrics. 2004. V. 59. № 1. P. 171.

2. Крижановская О.О., Синяева Л.А., Карпов С.И. идр. // Сорбционные и хроматографические процессы. 2014. Т. 14. Вып. 5. С. 784.

3. Lee J., Durst R.W., Wrolstad R.E. // J. AOAC Internat. 2005. V. 88. № 5. Р. 1269.

4. Дейнека В.И., Хлебников В.А., Чулков А.Н. и др. // Химия растительного сырья. 2007. № 2. С. 63.

5. Дейнека Л.А., Чулков А.Н., Дейнека В.И. // Журн. прикл. химии. 2009. № 5. С. 742.

6. Sidorov A., Deineka V., Kostenko M. et al. // Res. J. Pharm. Biol. Chem. Sci. 2016. V. 7. № 5. P. 2318.

7. Дейнека Л.А., Блинова И.П., Чулков А.Н. идр. // Научн. ведомости БелГУ. С. Медицина. Фармация. 2012. № 10 (129). Вып. 18/2. С. 60.

8. Deineka L., Deineka V., Tokhtar V. et al. // Res. J. Pharm. Biol. Chem. Sci. 2015. V. 6. № 5. P. 944. 